\title{
Perceptions and plans of Canadian food and beverage businesses regarding cannabis as a food ingredient.
}

\author{
Sylvain Charlebois ${ }^{1 *}$, Brian Sterling ${ }^{2}$ \\ ${ }^{1}$ Faculty of Management, Dalhousie University, Halifax, Nova Scotia, Canada \\ ${ }^{2}$ SCS Consulting, Oakville, Ontario, Canada
}

\begin{abstract}
Cannabis-infused consumables may become a fact of life in Canada by the end of 2019 and, according to this study, food companies are already seriously considering their options for entering the cannabis-infused consumables market. Food and beverage businesses continuously search for growth; new products using cannabis as an ingredient are seen by many as a fresh market that is going to open significant opportunities for growth and earnings. This study does not look at the functional effects of cannabis, but rather food processors' perceptions and plans regarding cannabis as a food ingredient once it is legalized. It explores several dimensions, including perceived risks associated with this embryonic sector, what those risks specifically might be, and any sense of competitive urgency that they may feel. Combined with a similar examination of Canadian consumer attitudes to cannabis consumables, published in January 2018, the two reports provide a unique view of the potential opportunities, hazards, and impediments in this new market sector. A total of 294 food and beverage firms were surveyed in August 2018. Just under $40 \%$ of these organizations say they support legalization of cannabisinfused products, while a substantial minority $(41 \%)$ are ambivalent. Over $65 \%$ of responding companies are concerned about the risks edibles represent to children and young adults. That said, $16.4 \%$ of surveyed companies confirmed that they are either planning to launch a product as soon as edibles are legal, or are already serving a market with such a product. The most commonly stated reason for not entering the cannabis consumables market is that cannabis is not compatible with their current product line. A general lack of understanding of cannabinoids was the second most popular reason given. The results highlight how just risk-adverse Canadian food industry leaders currently are regarding cannabis. Liability risks $(47.1 \%)$ and reputational risks $(20.1 \%)$ are cited as significant deterrents to entering the cannabis-infused consumables market. Other hazards include the lack of regulatory clarity, training of front-line staff, the residual social stigma of cannabis itself, questions about supply chain reliability, and the need for different, tamper-proof packaging. Plainly, regulatory questions remain top-of-mind for most company officials. Food and beverage firms say they are awaiting regulatory guidance as a condition of their plans. Respondents also state that governments are likely the key resource that they would employ during product and strategy development. More than $30 \%$ of respondents say governments are their primary choice, followed by their own internal resources. And $\mathbf{4 5 . 5 \%}$ see government bearing the chief responsibility for addressing cannabis-infused edibles issues. At the time of this writing, Health Canada has said it expects to begin industry consultations concerning cannabis consumables early in 2019. Until then, based on this survey's results, we perhaps should expect enthusiastic, yet limited, interest related to these products.
\end{abstract}

Keywords: Food ingredient, Cannabis

\section{Introduction}

In the $21^{\text {st }}$ century, economic growth in developed countries has led to rising demand for products that go beyond basic needs and seek to satisfy rapidly shifting wants and desires. Coincident with this growth, social and environmental awareness has increased, creating higher demand for sustainable, healthy food and an opportunity for food companies to add value through innovation in new products $[1,2]$. Innovation can be driven by the inclusion of specific ingredients, as we have seen in the development of functional foods in recent years. Changes to the quality and fabric of our food supply are often inspired by market-based research which captures consumer sentiment related to innovative ideas for new foods.

However, little empirical work has been done to assess how food processors perceive food innovation in cases where a longstanding illicit ingredient, if made legal (cannabis in this case), is incorporated into a food or drink. Cannabis, specifically nonmedicinal (recreational) cannabis, is the subject of this study. 
At the time of this study, the Canadian government has legalized dried flower, seeds, and oils for use as recreational cannabis (also known as non-medicinal cannabis or cannabis for adult-use). At the same time, a national regulatory framework for cannabis-infused consumables (so-called edibles) has yet to be published or discussed with the food and beverage industry. And the timeline for consultations on these regulations remains unclear.

Canada is the first G7 nation to make recreational cannabis legal. Uruguay, in 2014, became the first country to legalize the sale and distribution of cannabis nationally. California, Washington State and Colorado are just three of 9 US states that have legalized the recreational use of cannabis. Many industrialized countries are considering making cannabis legal for recreational purposes. In more than 11 European countries, including the Netherlands, United Kingdom, Belgium, and Spain, cannabis is legalized for medicinal use or is decriminalised. Australia joined the list of countries where medicinal cannabis became legal in 2016. Canada legalized medical cannabis in 2001, although accessibility was restricted and highly regulated through Health Canada until recreational use became nationally legalized in October 2018.

Cannabis is the most widely used illicit drug in the developed world and its use has been associated with negative social and economic outcomes [3]. It has been estimated that over 170 million people world-wide consume cannabis daily [4]. However, now the challenge for policymakers and public health officials is to navigate through a myriad of possibilities resulting from the widespread consumption of cannabis by a larger portion of the population.

By comparing the results of the previous consumer perception survey against this newest one, we note the close parallels between the issues and a confluence of concerns from consumers and food/beverage companies [1]. While this observation in itself may not be especially astonishing, it does insinuate conceivable development of a working framework in which concerns of the consuming public and of business (Figure 1) are addressed collaboratively.
An intriguing possibility exists in which the principles around Maslow's hierarchy of needs [5] may be applied to better understand the comparative needs of consumers, regulators, and businesses. This can be achieved by explicitly utilizing Maslow's concepts and principles around the 'basic' and 'psychological' needs that are their motivations. These needs that lower down in the hierarchy must be largely satisfied before individuals can attend to needs that are considered higher up. From the bottom of Maslow's model upwards: Basic needs are physiological and safety based; Psychological needs include affiliation, love, and esteem; Self-actualization needs (the highest order in Maslow's hierarchy), which are unlikely to play a large role for groups of stakeholders in this discussion, yet may become powerful motivation once other needs are met. Further consultations and dialogue on stakeholder concerns may enable them to be addressed concurrently.

This survey assesses both the risks and plans that food and drink manufacturers may perceive in a market in which a formerly-illicit product becomes readily available for general public consumption. In our later discussion we will revisit the parallels between the perceived risks (as seen by consumers and businesses) with cannabis-infused consumables and Maslow's essential hierarchy of needs.

Finally, this report presents limitations and future paths for further research.

\section{Pragmatic Considerations}

Cannabis, is the most widely used drug in Western societies and globally has one of the longest recorded histories of human use. The popularity of cannabis as a recreational drug is due to its ability to alter sensory perception, relieve pain, and cause elation and euphoria. The recent (1988) identification of cannabinoid receptors and their endogenous lipid ligands has triggered an explosive growth in studies of the endocannabinoid system and its regulatory functions in health and disease [6].

Absorption, distribution, and metabolism determine the onset and duration of action of each dosage format. Absorption has the most variability, and is affected by product lipophilicity,

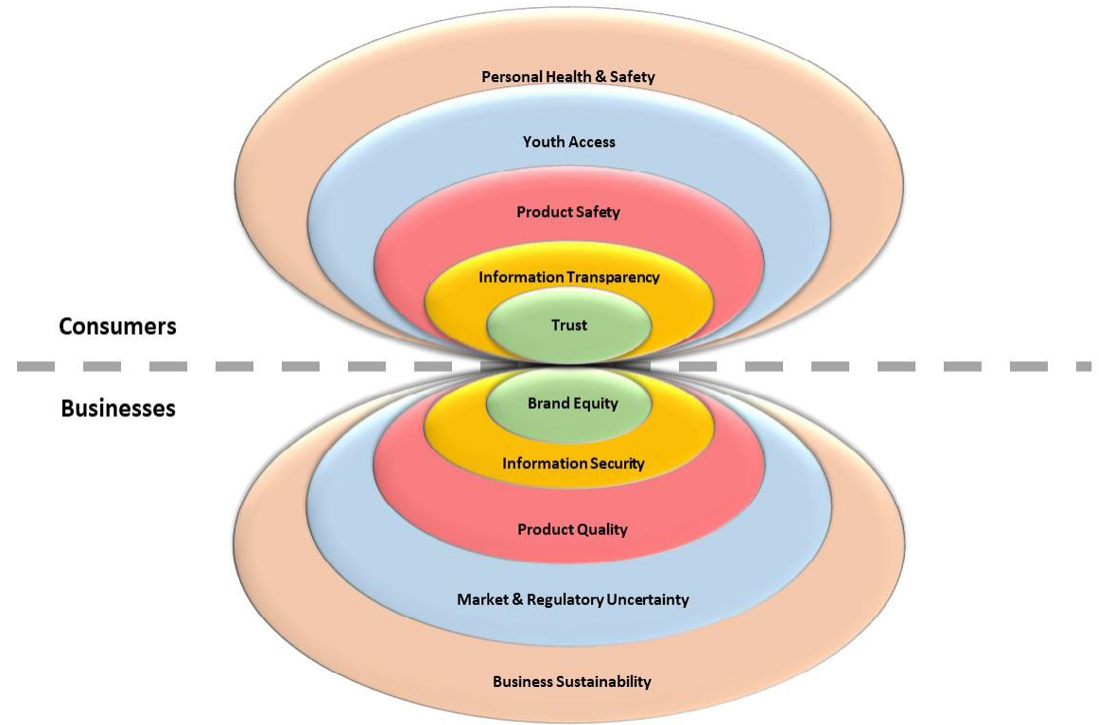

Figure 1. Consuming public and of business. 
bioavailability as well as the inherent organ tissue differences (i.e., alveolar, dermal vs. gastric). Cannabinoids are lipophilic and have low water solubility. Therefore, for topical or oral routes, they are best absorbed in the presence of fat, oils or polar solvents, such as ethanol. There is suggestion that newer technology such as using nano- or ionized particles or the use omega fats in carrier oil can enhance absorption; or for topical preparations, using ingredients to mildly disrupt the skin barrier may allow greater absorption of active ingredient. Factors such as recent meals, depth of inhalation, duration of breath holding, temperature of vaporizer all affect cannabis absorption, which can vary from $20 \%-30 \%$ for ingestion, up to $10-60 \%$ for inhalation [7].

Food and beverage businesses continuously seek growth, and new products using cannabis ingredients are seen by many as a brand new market that is going to open new opportunities for sales growth and earnings [8].

Consumers increasingly prefer consumable cannabis products to smoking cannabis because there is no smell from smoke and no second-hand smoke. Other reasons participants like edibles included convenience, discreetness, longer-lasting effects, less intense effects, and edibles' ability to aid in relaxation and reduce anxiety more so than smoking cannabis. Concerns and dislikes about consumable products include delayed effects, unexpected highs, the unpredictability of the high, and inconsistency of distribution of marijuana in the product [9].

There are thousands of individual cannabis types, which patients and purveyors may erroneously refer to as 'strains', whereas the preferred term is chemical variety or 'chemovar'. Each chemovar contains varying concentrations of cannabinoids and other components with important pharmacological and modulatory effects include the mono-terpenoids myrcene (analgesic, sedating), limonene (antidepressant and immune-stimulating), pinene (acetylcholinesterase inhibitor alleviating short-term memory impairment from THC) and the sesquiterpenoid beta-caryophyllene (anti-inflammatory analgesic). The relative proportions of these and other components are the primary determinants of the pharmacological effects and adverse events associated with a particular cannabis chemovar, and is critical information that should be available to patients and physicians recommending such treatment [10].

The manner in which cannabis is taken is not routinely measured in population-based surveys [11]. This is understandable considering the prohibition of cannabis and the historical predominance of smoking as the mode of consumption. However, given recent trends, it is becoming more important to for businesses to assess alternative modes of consumption so they have a more complete picture of the nature of marijuana use.

Consumable alternatives to inhaled cannabis (i.e., cannabisinfused food, drinks, candies, etc.), have become increasingly popular. For instance, cannabis consumable products accounted for approximately $40 \%$ of the estimated $\$ 700$ million in medical and recreational cannabis sales in the (US legalization) state of Colorado in 2014 [12]. Among cannabis-using adults in a representative US survey, $16 \%$ reported cannabis edible or drinkable product use [13]. Edible products are typically more popular than drinkables, as $49 \%$ of a medical cannabis user sample $(n=128)$ in Australia reported cannabis edibles use, whereas only $5 \%$ reported drinkable use [14].

\section{Methodology}

This study is derived from a quantitative analysis of data from an online survey of those working with food and beverage companies, aged 18 and over, living in Canada for at least twelve months. The survey instrument was divided into three parts.

The first part included questions related to perceptions of manufacturing cannabis-infused consumable products. Respondents were asked if they in favour of legalization of cannabis edibles in general. We were seeking to understand if there are correlations with other developments which their companies pursue and to compare this survey data with that from consumer perception surveys. The second section asked about perceived risks and barriers associated with manufacturing cannabis consumables. This part probes for concerns related to the business risks in making and selling these products, gaps in knowledge about cannabis and its effects, and on what resources they intend to rely to mitigate the risks and gaps.

In the last section, we asked questions about company type, size, and sector, geographic location (province), the person's role in the company, official positions, and general level of food safety discipline to assess these characteristics.

The survey was administered online during the month of August 2018. Using an approach consistent with similar studies on food consumption and trends the survey was distributed (in French and English) across Canada using Qualtrics, targeting several different regions $[15,16]$. Invitations were also posted on social media outlets and respondents were sought through an open invitation. Results were monitored regularly to help ensure broad representation of Canadian food and beverage businesses, and modifications were made in recruitment among underrepresented demographics.

Regions measured were the Atlantic Region, Québec, Ontario, the Prairies, and British Columbia (BC). These geographical locations for the survey were chosen to maximise regional and socioeconomic variability and to maintain similarity with previous consumer attitude surveys. The sample size of 294 was considered adequate for the purposes of this exploratory study. Each respondent took an average of just over 5 minutes to complete the survey. The completion rate of the survey was $84 \%$, which was based on the number of people who started and completed the survey; this is relatively high for surveys of this type [17-19].

\section{Results}

Of all businesses surveyed, a total of $39 \%$ either agree or strongly agree with national legalization of cannabis-infused consumables (or sometimes called, edibles). Nearly the same number of businesses $(41 \%)$ say they are neither for nor against legalization. The survey also highlights a significant majority of companies $(65 \%)$ are concerned about the risks related to children having access to cannabis consumables. These results suggest that most food and beverage firms understand the need 
to address the risks for children and young adults who may gain access to a psychoactive drug through these products, once they are in the market.

Health perceptions related to cannabis consumables were included in the survey and it is apparent that this is an area where food and beverage processors may need to learn more about the business potential of these products. On questions related to perceptions of what drivers might cause consumers to ingest cannabis-infused products, the survey generated interesting results. Just over 1 in five companies (23.6\%) say that they think consumers will consider cannabis ingredients as 'healthy'; a larger minority of firms (40.3\%) disagree or strongly disagree at this time that health is a key driver of consumer demand for cannabis edibles.

Previously and based on anecdotal remarks from executives, the authors have suggested that Canadian food and drink companies are sitting on the sidelines when it comes to cannabis. The survey confirms this, with only $14 \%$ expressing a sense of urgency in investigating cannabis-infused products; only $2 \%$ said they are already making edibles (for other markets). More than $41 \%$ say they have no plans to do look into cannabis infusions, and roughly $20 \%$ of companies say they may explore cannabis edibles but there is no urgency.

Using forced-field selection, a significant minority of companies $(30 \%)$ say they will rely on government resources first when it comes to developing new products and/or business strategies on cannabis-infused consumables. This aligns with observations that regulatory questions are top-of-mind for food and beverage executives who may be considering this market. Internal company resources are considered important by $26.4 \%$ of respondents; this is followed closely by specialized consultants $(25.7 \%)$. These resources are the dominant ones, with academia and industry associations following well behind at $10.7 \%$ and $7.1 \%$ respectively.

This reliance on government resources needs to be taken in the context of an (as yet) undefined and largely unregulated market; it seems intuitively consistent that companies would look first for help from regulators and other government agencies for assistance. The survey results bear out this intuition: fully $45.5 \%$ of companies say that governments bear the primary responsibility for cannabis and/or consumables issues. Only $20 \%$ say they see themselves (food and beverage processors) as primarily responsible, and nearly 1 in five (17\%) are unclear who has this responsibility. Other responses to this question include ingredient suppliers $(12 \%)$ and retailer/food service firms $(4.5 \%)$.

When asked to look into the future, respondents provide varying opinions on where they thought issues regarding cannabis and consumables would most likely originate. The replies seem to betray a mindset that is fixed in the current market: $22 \%$ think that most problems will occur in private homes; $18.6 \%$ say it will be at retail food stores, while about $17 \%$ say food processing facilities. Another $17 \%$ say it will be in food service operations, and $9 \%$ think it will be at the licensed producer (grower) level. Only 15\% were unsure or had no opinion.
Clearly companies are preoccupied with the risks associated with getting into the cannabis-infused consumables market. Several questions were asked on this topic. The survey first asked companies to list the top 3 barriers or constraints that will influence the presence of cannabis edibles in the food and beverage market. Interestingly, social stigma was consistently ranked first by respondents. This is a residual behavioral and cultural factor over which most food and drink companies will have little or no initial control. Awareness and education will take time to gain traction with a generally naïve public.

The next most commonly cited factors that companies say will inhibit cannabis-infused consumables, in order, are:

- Lack of regulatory clarity

- Lack of education about cannabis

- $\quad$ Misinformation (possibly linked with the above)

- Lack of retail options

- $\quad$ Training of their own staff

- $\quad$ Negative health consequences

- $\quad$ Supply chain reliability

- Ingredient control

- $\quad$ Theft

- Tamperproof packaging.

When asked about the impact of adding cannabis to their product lines, a substantial minority $(45.5 \%)$ say that it would increase their business risks, while one-third (32.8\%) say it will significantly raise their risks. Only $19 \%$ of respondents say that it would not change their risks. Interestingly, a small number (3\%) say it would decrease their business risks.

The survey then plunged deeper into the question of risk by asking respondents to rate the importance of 4 key areas of risk. The results are instructive (Figure 2):

- $47 \%$ say liability risks are their number 1 concern misuse of their products with negative consequences;

- $20 \%$ say reputational (consumer perception) risks are their primary worry;

- $17 \%$ say operational risks are most important - not having proper processes and procedures in place for cannabis; and

- $16 \%$ say that their first concern is the associative risk to their brand.

When it comes to their subsequent ranking of risks, $36 \%$ say that operational risks are $\# 2$, and $27 \%$ say their second highest concern is reputational risks. The second ranked risks continue with just over $22 \%$ saying liability risks, and $14 \%$ pointing at brand risks

Just as instructive were the responses on how companies plan to manage these 4 areas of risk (more than one response could be given):

- $\quad$ Nearly 7 in ten $(69 \%)$ say they intend to address 

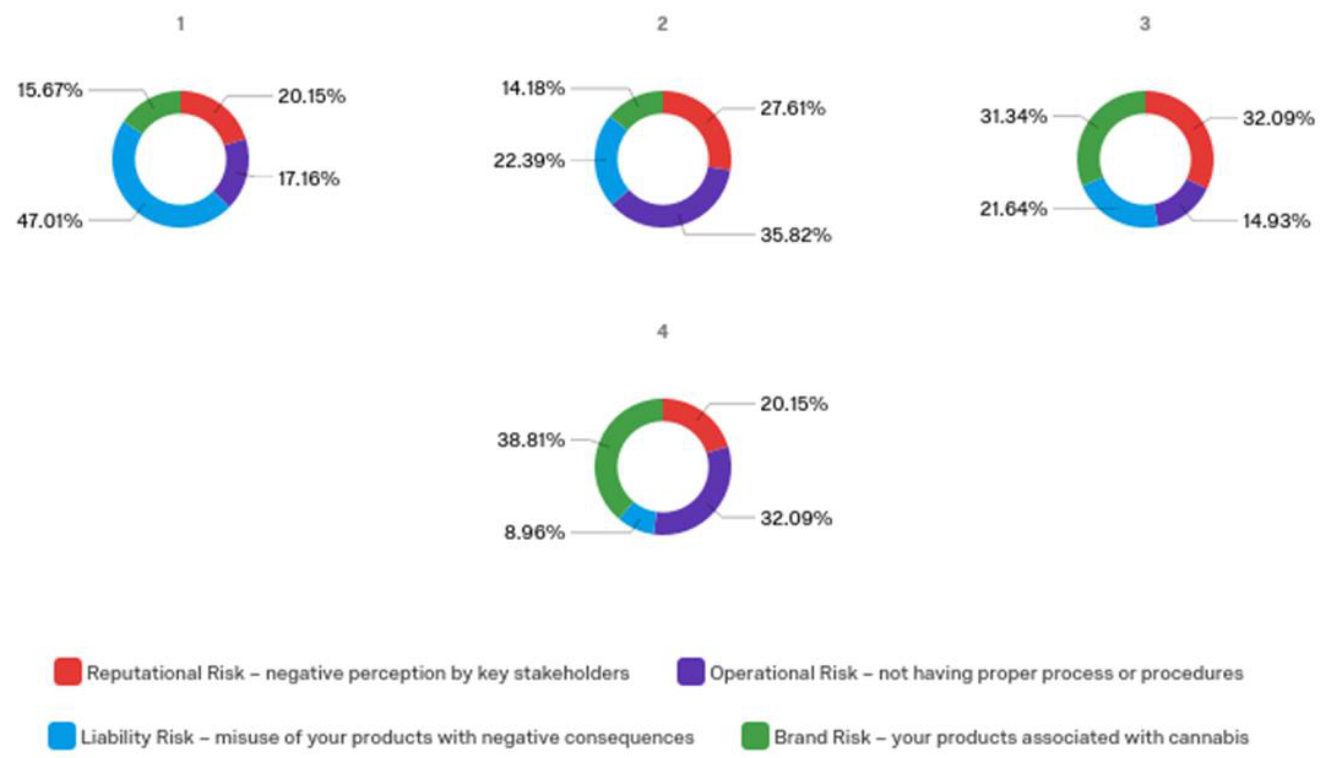

Figure 2. 4 key areas of risks.

these risks with existing internal resources, augmented with additional internal processes

- $44 \%$ say that they intent to manage these risks by outsourcing the necessary resources and processes (for example co-manufacturing)

- Another $44 \%$ say they will boost their insurance to manage the risks

- Over 30\% claim they will use existing resources and process only.

\section{Discussion}

The results offer mixed messages about how food and beverage companies view legalization of recreational cannabis and its use in consumables. The survey generally found there is substantial stigma and confusion about what cannabis-infused products would mean to their organizations and to consumer preferences. We infer from the data that executives from larger companies do not want to expose their brand or company to perceived hazards with cannabis. Whether or not science shows cannabis to be relatively harmless compared to other intoxicants, a sense of social stigma remains a strongly held one by these respondents.

While a large minority (39\%) of firms are favourable to legalization of cannabis, they also reflect the same concerns about risks to children as previous studies of consumer preferences and perceptions. The results also show that food and beverage companies do not think consumers see cannabis as a healthy ingredient or that they would include in a healthy dietary regime. This is consistent with consumer attitudes too [1].

In the case of cannabis, curiosity seems to be a substantial driver that may attract consumers to food products, whether it is at a retail store or in a restaurant. The effects one would experience by consuming cannabis is of interest too, but the data do not show whether this would be a long-term driver of consumption. This curiosity magnifies the concerns that cannabis-infused food products could become readily accessible by children. There is clearly an opportunity for the industry to educate consumers, and for regulators to respond to these concerns so that the risk is mitigated.

At the time this study was conducted, Health Canada was preparing draft regulations related to the production and distribution of cannabis-infused products, with regulations for consumable products to be completed within twelve months of the official legalization date. Regardless of what specific regulations may be developed, in this survey a majority of food and beverage companies say they look to governments first when it comes to addressing issues relating to cannabis consumables, as well as mitigating the risks of harm to consumers. Industry leadership and use of internal resources are seen as secondary resources, although the regulatory waiting period will likely encourage input and feedback from the industry.

The regulatory framework for cannabis-infused products will be unclear for at least the next several months (if not longer), which opens businesses to attendant risks. Understanding that a regulatory regime still must first be created, most food and drink businesses are focused on the business risks associated with introducing cannabis into their product lines. While liability is of primary concern, companies are also worried about reputational risks to key stakeholders and potential risks to their brand. We foresee this will lead to many food and beverage firms building new brands and promotions, and possibly co-manufacturing with third parties, to mitigate the impact on their established businesses and brands. The industry should also leverage lessons learned by US-based food and beverage competitors that have already entered the market.

Clearly companies foresee that this is not just another novelty or interesting flavour ingredient. Given the nature of the market, and an increasingly competitive business environment, food and beverage firms see cannabis as a new way to grow; cannabis represents a potent path to increase revenues and margins. Given the perceived benefits and risks suggested by this and other studies, the food industry, including retail and 
food service, is wise to proceed with caution. Caution is good; timidity, probably not so much.

There remains a good deal of confusion about whether cannabis is a healthy dietary option, and imprecise concerns about transparency of information and potency of products. Consumers of edible products may generally be even less informed than those who currently smoke or vaporize cannabis. All of these factors make it challenging for policy makers and manufacturers to develop and position edible cannabis products in the market. At the very least, it suggests that there is work to do in educating consumers and building trust.

Two strengths of this study are: the recruitment of a national sample from various food and beverage businesses within Canada, and the parallels between this study and the previously cited survey of consumers. Limitations are that the survey is not fully representative of all Canadian food and drink companies, and cannot be compared with other countries due to methodological differences that are likely to be present.

In addition, this survey primarily assessed perceptions based on an assumption that use of cannabis in consumable products will fall under regulation in a year. This may happen as currently planned; however, anticipating what governments may or may not do is a dicey pursuit. Despite these limitations, the exploratory nature of this study provides valuable insights as a foundation for future research on how cannabis would be used as a food ingredient.

From a policy perspective, more work is required. It is clear that both provincial and federal governments see the legalization of recreational cannabis as a new source of tax revenue; however, risks associated with cannabis as a food ingredient are varied and compelling to many food and drink companies. With any regulatory framework, the science of risks must be examined; this holds especially true of cannabis-infused consumables.

Better understanding of the risks and benefits of cannabis food and drink products should be clearly communicated through numerous channels, including public and professional education. This is particularly applicable for the risks posed to children and/or young adults. Trade groups and the cannabis industry may be aware of these risks, but there has been little or no communication, nor any guidelines, given by public authorities on this matter [18]. As seen in some US states, this can lead to short-term, opportunistic decisions by individual businesses, potentially increasing risks for consumers and society as a whole.
Revisiting Figure 1 in light of a conceivable link with Maslow's theory, we then posit that the two key groups pertinent to this discussion or risks (consumers and companies) may demonstrate 'satisfaction' based on perceived risks and the motivations and behaviours similar to Table 1.

For example, physiological needs are primary or fundamental requirements for survival according to Maslow. In the consumer perception survey [1], respondents said that personal health and safety considerations were critical; specifically consumers want to know that their food and drinks are safe to ingest. This is tightly followed by concerns about children and youths being able to access cannabis, which are a second-order needs in Maslow's hierarchy. These requirements must be met, along with the next concern: product safety. Often consumers will rely on information (good and bad) to make a decision on their satisfaction with how a business meets these needs. Therefore, information transparency is a logical next level need; transparency helps support or weaken a consumer's necessary access to data. If it is lacking, then the need for trust is never fully satisfied. At best, a company may win contingent trust.

For a business, there may not be 'physiological' needs in a biological sense; however, there are primary or fundamental requirements just as critical for business sustainability. These are often defined as managing the triple bottom line - a company's financial, social and environmental risks, and its obligations and opportunities. These three are sometimes referred to as profits, people and planet [19]. It is apparent then how business sustainability can seem a 'physiological need'; the survival of a business depends in it. Continuing the comparison, companies clearly feel that their concern about business sustainability is closely linked to questions about the market and regulatory landscape. These are the 'safety needs' that a company can typically reference when mitigating risks associated with an unknown market and consumer base.

We could continue the analogy; however the point is that in these earliest stages of market and regulatory development in the Canadian cannabis and food and beverage industries, there is a strong parallel, if not alignment, of business and public needs. At the moment, the concerns and issues of the customary adversaries in such dialogues are notably consistent.

If leaders of industry, consumers, and regulatory agencies can recognize this subtle affiliation of needs, there is a unique opportunity to accelerate the pace of development of regulations and of a robust, new sector in the food and beverage industry.

Table 1. Demonstrate 'satisfaction' based on perceived risks and Maslow's Hierarchy.

\begin{tabular}{|l|l|l|}
\hline \multicolumn{1}{|c|}{ Perceived Risk (Survey Results) } & \multicolumn{1}{c|}{ Maslow's Hierarchy of Needs } \\
\hline \multirow{3}{*}{ Consumers } & Personal Health \& Safety & Physiological - biological requirements for human survival \\
\hline & Youth Access & Safety - protection from elements, security, order, law, stability, freedom from fear. \\
\hline & Product Safety & Safety - protection from elements, security, order, law, stability, freedom from fear. \\
\hline & Information Transparency & Affiliation - social sense of belongingness and the need for interpersonal relationships \\
\hline & Trust & Esteem - sense of endorsement or confidence in others \\
\hline Business Sustainability & Physiological - fundamental requirements for business survival \\
\hline Business & Market \& Regulatory Uncertainty & Safety - protection from elements, security, order, law, stability, freedom from fear. \\
\hline & Product Quality & Safety - protection from elements, security, order, law, stability, freedom from fear. \\
\hline & Information Security & Affiliation - social license, a sense of belongingness and the need for legitimacy to operate. \\
\hline & Brand Equity & Esteem - the strength of reputation or respect from customers for the business \\
\hline
\end{tabular}




\section{Conclusion}

The results of the survey point to a need for broader public dialogue (perhaps most with consumers and front-line workers) on cannabis and cannabinoids used in food and drink. More research is required to better appreciate the specific biological, sociological, and economic dimensions of incorporating cannabis into consumable products.

Most importantly, both businesses and consumers express concerns that cannabis-infused consumables could increase the risk to personal safety and the risk of exposure to cannabinoids by children and youths. Additional scientific research is needed to quantify the risk-benefit balance of cannabis-infused consumable products, so that health authorities can conduct well-informed discussions with medical professionals, food businesses, and consumers.

At the time of this writing, Health Canada has said it expects to begin industry consultations concerning cannabis consumables early in 2019. Until then, based on this survey's results, we perhaps should expect enthusiastic, yet limited, interest related to these products.

\section{References}

1. Charlebois S, Simogyi S, Sterling B. Marijuana-infused food and Canadian consumers' willingness to consider "recreational" marijuana as a food ingredient. Trends in Food Science \& Technology 2018.

2. Dutra de Barcellos M, Thøgersen J, Gattermann Perin M. Consumer buying motives and attitudes towards organic food in two emerging markets: China and Brazil. International Marketing Review. 2015;32(3/4):389-413.

3. Hartman M, Sreeram P, Wilson M. Rapid Synthesis: Identifying the impacts of cannabis legalization on youth, and the responses that can be taken by public institutions. McMaster Health Forum, April 2017.

4. Patel S, Cone R. A cellular basis for the munchies. Nature, International Journal of Science. 2015;519: 38-40.

5. Maslow, Abraham H, Frager R. Motivation and personality. Book. 1987. New York: Harper and Row.

6. Pacher P, Batkai S, Kunos G. The endocannabinoid system as an emerging target of pharmacotherapy. Pharmacological Reviews. 2006;58:389-462.

7. Huestis M. Human Cannabinoid Pharmacokinetics. Chem Biodivers.2007;4:1770-804.

8. https://business.financialpost.com/cannabis/cannabisbusiness/coca-cola-is-eyeing-the-cannabismarket

9. Giombi K, Kosa K, Rains C, et al. Consumers' Perceptions of Edible Marijuana Products for Recreational Use: Likes, Dislikes, and Reasons for Use, Substance Use \& Misuse. 2018;53(4):541-7.

10. MacCallum C, Russo E. Practical considerations in medical cannabis administration and dosing. European Journal of Internal Medicine. January 2018.

11. Johnson R, Brooks-Russell A, Ma M, et al. Usual Modes of Marijuana Consumption Among High School Students in Colorado. 2016;77(4):580-8.

12. Weis S. Edibles: For experts only? Ingesting marijuana, as opposed to smoking it, has come a long way since the days of homemade pot brownies. State Legislatures. 2015; 41:23

13. Schauer GL, King BA, Bunnell RE, et al. Toking, vaping, and eating for health or fun: Marijuana use patterns in adults, U.S., 2014. American Journal of Preventive Medicine. 2016;50(1):1-8

14. Swift W, Gates P, Dillon P. Survey of Australians using cannabis for medical purposes. Harm Reduction Journal. 2005.

15. Beardsworth A, Bryman A. Meat consumption and meat avoidance among young people: An 11- year longitudinal study. British Food Journal. 2004:106(4);313-27, https:// doi.org/10.1108/00070700410529573

16. Redmond E, Griffith C. Consumer Food Handling in the Home: A Review of Food Safety Studies. Journal of Food Protection. 2003;66(1);130-61

17. LaRose R, Tsai HS. Completion rates and non-response error in online surveys: Comparing sweepstakes and pre-paid cash incentives in studies of online behavior. Computers in Human Behavior. 2014;34:110-9.

18. Charlebois, S, Summan A. A Risk Communication Model for Food Regulatory Agencies in Modern Society. Trends in Food Science \& Technology, August 2015.

19. Financial Times lexicon. http://lexicon.ft.com/Term?term=businesssustainability

\section{*Correspondence to:}

Sylvain Charlebois

Kenneth C. Rowe Management Building,

Room 3059, 6100 University Avenue

PO Box 15000, Halifax NS

Canada

Tel: 902-494-7822

E-mail: sylvain.charlebois@dal.ca 